\title{
Infliximab in ulcerative colitis
}

\author{
Avi Levin \\ Oren Shibolet \\ Gastroenterology and Liver Units, \\ Division of Medicine, Hadassah- \\ Hebrew University Medical center, \\ Jerusalem, Israel
}

\begin{abstract}
Infliximab, a monoclonal anti-TNF- $\alpha$ antibody, is commonly used for treatment of moderate to severe Crohn's disease (CD). Its role in the treatment for ulcerative colitis (UC) remains controversial. We review the role of TNF- $\alpha$ in the pathogenesis of UC and describe the randomized, double-blind, placebo-controlled trials and systematic reviews that assess the efficacy of infliximab in the treatment of moderate to severe UC.
\end{abstract}

Keywords: infliximab, ulcerative colitis, anti-TNF- $\alpha$ antibody, crohn's disease

\section{Introduction}

Ulcerative colitis (UC) is a chronic remitting and relapsing disease characterized by mucosal inflammation of the colon. The extent of disease varies from limited involvement of the rectum to involvement of the whole colon. Rarely, there is mild involvement of the distal part of the ileum, which is believed to be an "overflow" of inflammation from the cecum and is called backwash ileitis (Podolsky 2002).

UC manifests clinically as bloody diarrhea, abdominal pain, urgency, and tenesmus. In more severe cases, patients may develop systemic features, such as fever, tachycardia, nausea, vomiting, anorexia, weight loss, and an elevated CRP and erythrocyte sedimentation rate (Baumgart and Sandborn 2007). Clinically mild disease is defined as disease associated with fewer than 4 bowel movements a day, with or without blood in the stool and without systemic manifestations. In these patients, laboratory results are usually within the normal range. Moderate disease describes disease associated with more than 4 bowel movements a day with minimal systemic manifestations. Severe disease describes disease associated with more than 6 bowel movements a day, with blood in the stool and with systemic involvement (D'Haens et al 2007). The clinical course of UC is marked by exacerbations and remissions. Treatment of mild to moderate UC consists of oral and/or topical 5-aminosalicylates, oral and/or topical corticosteroids, and purine analogs (azathioprine and 6-mercaptopurine) (Carter et al 2004; Lichtenstein et al 2006). In $15 \%$ of the cases, the disease manifests as an episode of severe colitis necessitating hospitalization, administration of intravenous treatment, and sometimes colectomy. A small minority of the patients develop a frequently relapsing chronic disease (Binder 2004). Approximately $11 \%$ of the patients with UC develop extraintestinal manifestations over the course of their disease, which can parallel the activity of colitis or be independent of colonic disease. These manifestations include: small joint arthritis, sacroileitis, ankylosing spondylitis, erythema nodosum, pyoderma gangrenosum, apthous ulcers of the oral mucosa, episcleritis, uveitis, and sclerosing cholangitis (Danese et al 2005).

Early studies reported a $30 \%$ fatality rate for patients referred for a first attack of severe UC. However, as medical and surgical treatments improved, the mortality rates declined, and currently patients with UC have an overall normal life expectancy (Loftus et al 2000). Some reports even suggest decreased mortality in patients with UC compared with the general population (Winther et al 2003). Despite overall good 
survival rates, patients have an increased risk of colectomy, development of colorectal cancer (Soetikno et al 2002), and cholangiocarcinoma (Broome et al 1995).

Despite major advancements in medical therapy, treatment options for patients with moderate to severe UC remain limited. Steroid treatment was introduced in the 1950s and markedly reduced mortality in severe disease (Truelove and Witts 1955). The other remaining medical treatment option for patients with severe UC is the addition of cyclosporine. Although considered to be highly effective in inducing remission, double-blind, randomized, controlled trials assessing the efficacy of cyclosporine in this setting are limited (Lichtiger et al 1994; D'Haens et al 2001). Furthermore, cyclosporine has a narrow therapeutic index with potentially serious side effects, necessitating close monitoring of cyclosporine blood levels (Shibolet et al 2005). If treatment with steroids and cyclosporine fails to induce remission in severe UC, patients are faced with the need for colectomy. Even if patients respond to cyclosporine, most will eventually require colectomy (D'Haens et al 2001).

In the face of the paucity of treatment options it was essential to identify new therapies for moderate to severe UC that are easier to administer, have higher efficacy, are better tolerated and have a better safety profile.

The search for such drugs focused on elucidating the pathophysiology of inflammatory bowel disease (IBD) (Xavier and Podolsky 2007) and identifying possible drug targets (Korzenik and Podolsky 2006). Data from animal models of colitis and from human studies suggested that treatment with either anti-inflammatory cytokines or with pro-inflammatory cytokine blockers may modulate the course of IBD (Elson et al 2005). One of the major pro-inflammatory cytokines involved in the pathogenesis of IBD was discovered to be tumor necrosis factor- $\alpha$ (TNF- $\alpha$ ) (Papadakis and Targan 2000). This led to a breakthrough in IBD treatment with the introduction of anti-TNF- $\alpha$ antibody treatment that was initially used to treat Crohn's disease (Sandborn and Hanauer 1999).

\section{Pathogenesis of IBD}

Inflammatory bowel disease groups together two distinctive clinical entities: UC and CD. These two diseases differ in their location (colon only for UC vs the whole length of the intestinal tract for CD), pattern of distribution (continuous vs patchy), depth of involvement (mucosal vs transmural), and histology (crypt abscesses vs granulomas). It is therefore not surprising that their pathogenesis is considerably different. The pathogenesis of IBD has been hypothesized to be caused by an inappropriate immune response against luminal antigens in a genetically susceptible host resulting in uncontrolled intestinal inflammation (Xavier and Podolsky 2007). Inflammatory responses are traditionally classified into Th1 or Th2 responses based on the cytokine secretion profile of differentiated T lymphocytes. Both CD4+ and CD8+ T lymphocytes can be identified as either Th1 cells that produce IFN $\gamma$, IL-2, IL-12, and IL-18 or Th2 cells that secrete IL-4, IL-5, IL-6, IL-10, and IL-13. Recently, another type of immune response dubbed Th17 and characterized by production of IL-17 by specific IL-17 producing T lymphocytes has been described and was shown to play a role in the pathogenesis of IBD (Park et al 2005).

CD has been traditionally described as a Th1 mediated disease with the predominant cytokines being the pro-inflammatory cytokines IFN $\gamma$, IL-1 $\beta$, and IL-12. These cytokines contribute to an increase in mucosal permeability, collagen synthesis and recruitment of inflammatory cells. Most existing animal models of experimental colitis are skewed toward a Th1 response. These include trinitrobenzene (TNBs) colitis, dextran sodium sulfate (DSS) colitis, and the IL-10 knockout spontaneous colitis models. Another Th1 mediated granulomatous colitis model has been established by the adoptive transfer of normal CD45RBhigh T lymphocytes from Balb/C mice into SCID mice (Bouma and Strober 2003).

$\mathrm{UC}$, on the other hand, has been considered to be an atypical Th2 mediated disease characterized by CD4+ T lymphocytes bearing a natural killer (NK) T lymphocyte marker. Levels of IL-4, IL-5, IL-8, but also IL-1 $\beta$, IL-12, and IFN $\gamma$, are significantly higher in patients with UC than in healthy controls with a prominence of IL-4 and IL-5. Th2 predominant animal models of experimental colitis include the TCR $\alpha$ and WASP deficient mice, oxazolone colitis, and spontaneous colitis in cotton-top tamarins (Strober et al 2002). The recent discovery of Th17 cells and their role in IBD (Iwakura and Ishigame 2006) suggests that the Th1/Th2 hypothesis may represent an oversimplified model of this complex disease.

Despite the immunological and clinical differences between UC and CD, both diseases share part of their cytokine profile and exhibit elevated levels of TNF- $\alpha$.

\section{Tumor necrosis factor- $\alpha$}

TNF- $\alpha$ is a cytokine involved in inflammatory responses and is a member of the TNF-super-family. It is capable of killing tumor cells in vitro and causing hemorrhagic necrosis of transplantable tumors in mice (Old 1985; Bazzoni and Beulter 1996). TNF- $\alpha$ plays a role in multiple human 
disease processes including septic shock (Cauwels et al 2003), cancer/AIDS-associated cachexia (Argiles et al 1997), graft-versus-host disease (Jacobsohn and Vogelsang 2004), rheumatoid arthritis, and CD (Siddiqui and Scott 2005).

TNF- $\alpha$ is first produced as a $26-\mathrm{kDa}$ transmembrane protein, which can be cleaved by the metalloproteinasedesintegrin, TNF- $\alpha$ converting enzyme (TACE also known as ADAM17) to form a secreted, soluble $17-\mathrm{kDa}$ protein (Black et al 1997). Secreted TNF- $\alpha$ (sTNF- $\alpha$ ) aggregates into trimolecular complexes, which bind and activate the TNF receptors, TNF receptor type 1(TNFR1) and TNF receptor type 2 (TNFR2). TNFR1 is constitutively expressed in most tissues, and can be activated by both the membrane-bound and soluble forms of TNF- $\alpha$, while TNFR2 is expressed in cells of the immune system and responds to the membrane-bound form of TNF- $\alpha$. Following TNF- $\alpha$ binding, the receptors form trimmers and initiate intracellular signaling via 3 main pathways. Inflammatory signals are conveyed via NF- $\kappa$ B and MAPK pathways, while apoptotic signals are conveyed via TRADD, FADD and caspase- 8 (Vassalli 1992).

Monocytes/macrophages are the main source of TNF- $\alpha$, although $\mathrm{T}$ and $\mathrm{B}$ lymphocytes also produce significant amounts. Other cells known to produce TNF- $\alpha$ include NK cells, mast cells, Paneth cells, keratinocytes, astrocytes and microglial cells, smooth muscle cells, and certain tumor cell lines (Guy-Grand et al 1998; Nilsen et al 1998; Bischoff et al 1999). TNF- $\alpha$ is also produced by intestinal epithelial cells in response to bacterial invasion (Jung et al 1995).

\section{Infliximab}

Infliximab is a chimeric anti-TNF- $\alpha$ mouse monoclonal antibody (Baugh and Bucala 2001). Its Fab fragment consists of the mouse variable TNF binding region $(25 \%$ of the protein) and a human Fc fragment (75\% of the protein) (Elliott et al 1993; Knight et al 1993). The antibody binds both the soluble and transmembrane forms of TNF- $\alpha$ with high affinity and blocks their action (association constant $10^{10} / \mathrm{M}$ ), with a serum half-life of 10 days. It is administered intravenously at a dose of 5 or $10 \mathrm{mg} / \mathrm{kg}\left(\operatorname{Remicade}^{\circledR}\right)$. The temporal regimens employed in IBD include administration on weeks 0,2 , and 6 and then periodically every 8 weeks (Rutgeerts et al 2005).

The mechanism of action of infliximab is not clearly understood. Although initially thought to be mediated via neutralization of soluble TNF- $\alpha$, this is clearly not enough because treatment with other anti-TNF- $\alpha$ antibodies and soluble TNF- $\alpha$ receptors with similar or even greater neutralizing efficacy do not exert the same therapeutic effect (Remicade $^{\circledR}$; van Dullemen et al 1995; Hanauer et al 1998). Recent investigations suggest that the mechanism of action of TNF- $\alpha$-blocking agents is mediated via apoptosis of TNF$\alpha$-expressing inflammatory cells. These studies suggest that mucosal $\mathrm{T}$ cells of patients with IBD are highly resistant to apoptosis (Ina et al 1999; Atreya et al 2000). Resistance to apoptosis has been shown in both $\mathrm{CD}$ and $\mathrm{UC}$, but the mechanism behind this phenomenon seems to differ between the two conditions. The intrinsic defect in CD occurs in the mitochondrial pathway of apoptosis (imbalance of mitochondrial bcl-2/bax), whereas in UC it results from overexpression of FLICE-inhibitory protein (FLIP) and impairment of the caspase-mediated pathway of apoptosis (Peppelenbosch and van Deventer 2004). It was shown that infliximab can induce apoptosis of inflammatory cells including $\mathrm{T}$ cells and monocytes via a caspase-dependent mechanism. This effect was independent of $\mathrm{Fc} \gamma$-R binding or complement activation (Tilg et al 2007).

\section{Role of TNF- $\alpha$ in ulcerative colitis}

In the intestine, the direct effects of TNF- $\alpha$ on intestinal epithelium include disruption of the epithelial barrier, induction of apoptosis of epithelial cells, and secretion of chemokines from intestinal epithelial cells (Figure 1). TNF- $\alpha$ also activates the adaptive immune system of the bowel through recruitment and activation of neutrophils and macrophages (Guy-Grand et al 1998; Nilsen et al 1998; Bischoff et al 1999).

The role of TNF- $\alpha$ in the pathogenesis of IBD has been extensively studied and is convincingly established for CD (Plevy et al 1997; van Deventer 1997). In UC, the role of TNF has been less well characterized (Sands and Kaplan 2007). There are several examples of a genetic association between TNF- $\alpha$ gene polymorphism and susceptibility to $\mathrm{UC}$. The frequency of carriers for an increased TNF- $\alpha$ secretion polymorphism (-308A and -238G) was significantly increased in Japanese UC patients compared with healthy controls (Wilson et al 1993), while only weak association was observed in Chinese UC patients (Cao et al 2006). In contrast, in Dutch UC patients, the frequency of the same polymorphism site was markedly decreased (Bouma et al 1996, 1999). A positive transmission disequilibrium of another increased TNF- $\alpha$ secretion polymorphism (-857C) was shown in a UK UC Caucasian (van Heel et al 2002) patient population, while homozygosity for a TNF- $\alpha$ haplotype, (TNF- $\alpha,-1031 \mathrm{~T},-863 \mathrm{C},-857 \mathrm{C},-380 \mathrm{G},-308 \mathrm{G}$, $-238 \mathrm{G}$ ) associated with low TNF- $\alpha$ production was shown 


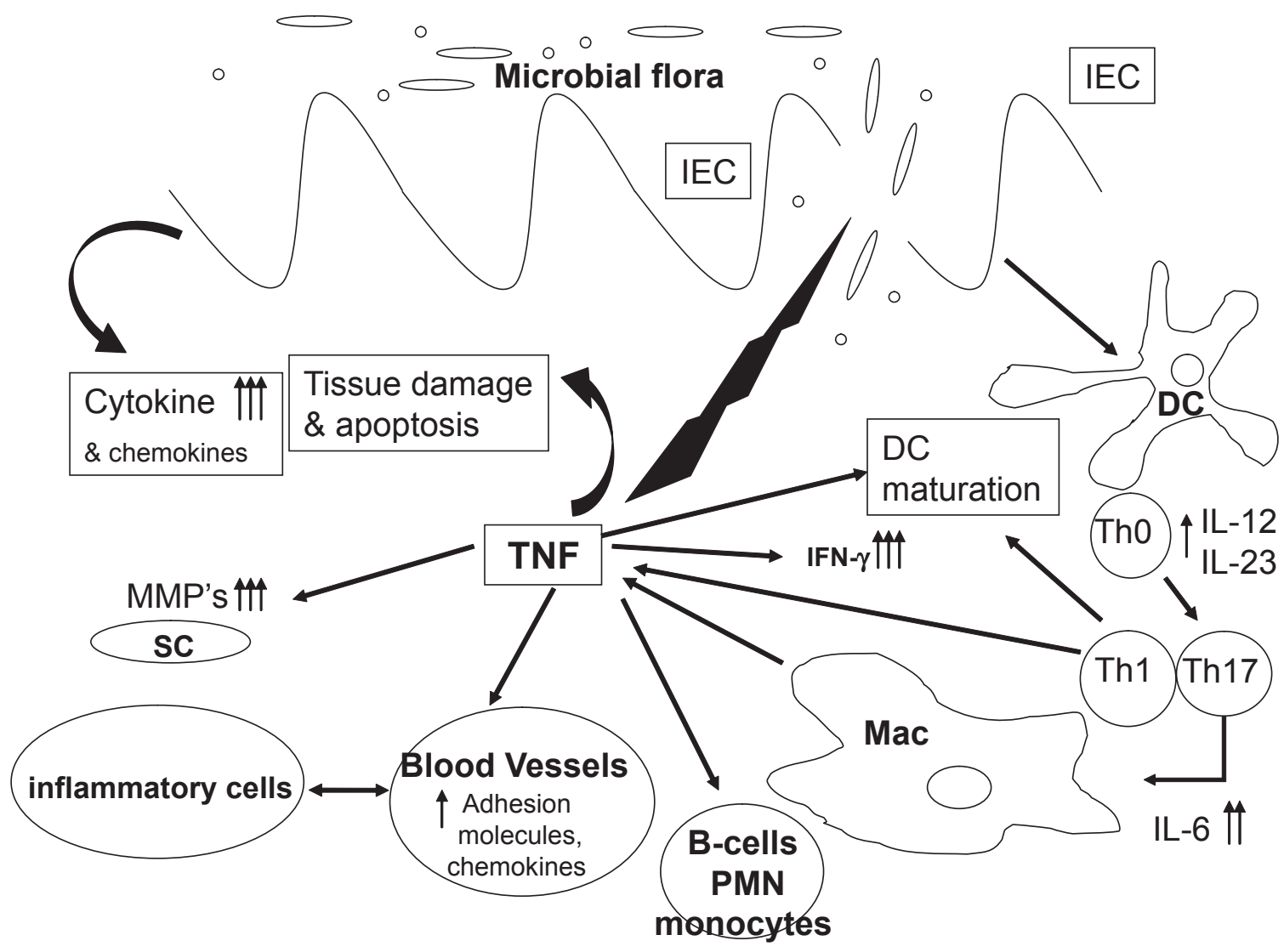

Figure I TNF- $\alpha$ involvement in intestinal inflammation. After initial damage to the mucosal barrier, TNF- $\alpha$ is secreted by T lymphocytes, macrophages (Mac), and intestinal epithelial cells causing epithelial cell apoptosis, production of cytokines and chemokines, maturation of DCs and activation of tissue metaloproteinases from SC.This in turn causes further barrier damage, activation of neutrophils (PMN) and B lymphocytes, up-regulation of adhesion molecules, and further recruitment of inflammatory cells. Abbreviations: IEC, intestinal epithelial cells; DC, dendritic cells; MMP, matrix metalloproteinase; SC, stromal cells.

to be more prevalent in UC patients with limited compared with extensive colitis (Ahmad et al 2003).

Several studies have shown significant increase in TNF- $\alpha$ in colonic mucosa of UC patients (MacDonald et al 1990; Murch et al 1993; Breese et al 1994). TNF- $\alpha$ production in the colon of UC patients was increased in inflamed but not in noninflamed areas (van Heel et al 2002). Similarly, TNF- $\alpha$ levels in stool samples from patients with active UC were significantly increased compared with healthy controls (Braegger et al 1992). Serum levels of TNF- $\alpha$ and TNFR were also shown to be significantly higher in UC patients than those of healthy controls and their levels were shown to correlate with disease activity (Komatsu et al 2001; Hanai et al 2004; Spoettl et al 2007).

In view of the data supporting the importance of TNF- $\alpha$ in the pathogenesis of UC and the role infliximab plays in inducing apoptosis, it was hypothesized that infliximab may exert a beneficial effect in the treatment of moderate to severe UC. Initially, anti-TNF- $\alpha$ treatment did not cause disease improvement in the experimental colitis Th2 type mouse model (oxazolone colitis) (Shen et al 2007). However, this model does not reliably mimic human disease. In cotton-top tamarins that develop spontaneous UC-like colitis, anti-TNF- $\alpha$ treatment markedly improved clinical condition (Watkins et al 1997). Therefore, studies were conducted to assess its efficacy in UC in humans. Although several open label studies assessed the role of infliximab in inducing remission in patients with moderate to severe UC (Chey et al 2001; Actis et al 2002; Kohn et al 2002; Su et al 2002; Castro Fernandez et al 2003; Gornet et al 2003; Armuzzi et al 2004; Bermejo et al 2004; Ljung et al 2004; Ochsenkuh et al 2004; Ruiz et al 2004; Kountouras et al 2005; Park et al 2005; Iwakura and Ishigame 2006), we chose to focus on randomized double-blind placebo-controlled studies and on systematic reviews and meta-analysis that assessed treatment efficacy.

\section{Double blind, placebo-controlled studies (see Table I)}

We identified 5 studies and 2 systematic reviews and metaanalyses that assessed the efficacy of infliximab in moderate to severe UC as compared to placebo. These studies used different outcome measures to assess their results. 
Table I Double blind, placebo-controlled studies of infliximab for UC

\begin{tabular}{|c|c|c|}
\hline & Reference & Outcome \\
\hline I & Järnerot et al 2005 & $\begin{array}{l}\text { Primary: Colectomy or } \\
\text { death within } 90 \text { days after } \\
\text { infusion. Secondary: clinical } \\
\text { remission according to the } \\
\text { Seo index and endoscopic } \\
\text { remission I and } 3 \text { months } \\
\text { after the infliximab/placebo } \\
\text { infusion }\end{array}$ \\
\hline
\end{tabular}

2 Probert et al 2003

4 Rutgeerts et al 2005 Similar to ACTI

5 Sands et al 200 I (ACT I) (ACT 2)

Disease activity 6 weeks after the first infusion using the UC scoring system. Two end points utilized to define remission: clinical remission UC scoring system $\leq 2$ and sigmoidoscopic remission as a Baron score of 0

Clinical response was defined as a decrease from baseline in the total Mayo score of at least 3 points with an accompanying decrease in the score for rectal bleeding of at least I point or an absolute score for rectal bleeding of 0 or I. Clinical remission was defined as a total Mayo score of 2 points or lower, with no individual score exceeding I point. Mucosal healing was defined as an absolute score for endoscopy of 0 or 1

Population
45 patients with an
acute moderate-
severe attack of UC
that did not respond
quickly to iv cortico-
steroids

Treatment protocol

Single infusion of infliximab/ placebo in dose of $5 \mathrm{mg} / \mathrm{kg}$

43 patients with UC who failed to respond to conventional treatment with glucocorticoids, and who were not in need of urgent colectomy

364 patients with moderate-to-severe active UC despite concurrent treatment with corticosteroids alone or in combination with azathioprine or 6-MP

364 patients with moderate-to-severe active ulcerative colitis despite concurrent treatment with corticosteroids alone or in combination with azathioprine or 6-MP and 5-ASA

II patients with severe active disease for at least 2 weeks despite of at least 5 days of corticosteroids
Infusions of infliximab $(5 \mathrm{mg} / \mathrm{kg}$ ) were given at weeks 0 and 2

Infusion of placebo or infliximab $(5 \mathrm{mg}$ or $10 \mathrm{mg} / \mathrm{kg}$ ) at weeks 0,2 , and 6 and then every 8 weeks through week 46

Infusion of placebo or infliximab $(5 \mathrm{mg}$ or $10 \mathrm{mg} / \mathrm{kg}$ ) at weeks 0,2 , and 6 and then every 8 weeks through week 22

Single infusion of infliximab at 5,10 , or $20 \mathrm{mg} / \mathrm{kg}$
Main result

7 (21\%) patients in the infliximab group and $14(67 \%)$ in the placebo group had a colectomy $(p=0.017)$ within 3 months after randomization

Six weeks after initiation of treatment, remission rates were $39 \%$ $(9 / 23)$ in infliximab group vs $30 \%$ $(6 / 20)$ in placebo $(\mathrm{p}=0.76)$ and a Baron score of $0(26 \%(6 / 23)$ vs $30 \%(6 / 20))$ respectively $(\mathrm{p}=0.96)$

$69 \%$ of patients who received $5 \mathrm{mg}$ of infliximab and $61 \%$ of those who received $10 \mathrm{mg}$ had a clinical response at week 8 compared with $37 \%$ of those who received placebo ( $\mathrm{p}<0.001$ for both comparisons with placebo). Mucosal healing at weeks 8,30 , and 54 occurred in significantly more patients in the infliximab groups than in the placebo groups

$64 \%$ and $69 \%$ of the patients in the 5 and $10 \mathrm{mg}$ infliximab groups respectively, had a clinical response at week 8 compared with $29 \%$ in the placebo $(p<0.001$ for both comparisons with placebo). Mucosal healing at weeks 8 and 30 occurred in significantly more patients in the infliximab groups than in the placebo groups

Four of 8 patients (50\%) who received infliximab were considered treatment successes at 2 weeks, compared $0 / 3$ patients who received placebo modified Truelove and Witts

severity score, 2) increase

in corticosteroid dosage, 3) addition of immunosuppressants, 4) colectomy, or 5) death 2 weeks after infusion. 
Additionally, various definitions were used to describe the main outcomes. For example, clinical remission has been defined as absence of active disease symptoms using Truelove and Witts criteria (the absence of blood and mucus in the stool and the absence of diarrhea), or the Lennard-Jones criteria (two or less bowel movements a day without other signs or symptoms) (Truelove and Witts 1955; Lennard-Jones et al 1975; Rutgeerts et al 2005).

It is important to understand that in contrast to $\mathrm{CD}$, in which the disease activity index (CDAI) is a well validated standardized tool in clinical trials, the outcome measures in UC are less well defined. Most trials use a combination of clinical, histological and endoscopic scores to describe remission. Others use combined scores which include Physician's global assessment (PGA) scores, ulcerative colitis disease activity index (UCDAI), Mayo score, a modified Lennard-Jones criteria, Rachmilewitz criteria, or Seo index. The lack of a standardized scoring system for UC makes comparison of trial results less accurate than comparing results in CD trials and makes interpretation of individual trial results prone to bias.

We identified a systematic review (Gisbert et al 2007), search date 2006, and a Cochrane review (Lawson et al 2006), search date 2005, that assessed the efficacy of infliximab in inducing remission in moderate to severe UC. Both identified 5 randomized, double-blind, placebo-controlled studies; however, while the systematic review by Gisbert et al reported the results in terms of short- and long-term response only, the Cochrane review by Lawson et al used a more complex approach of reporting on clinical remission and response, endoscopic and mucosal responses, and colectomy rates. We will describe the studies and the various differences between the two meta-analyses.

In the first double-blind, placebo-controlled trial, Sands et al (2001) recruited 11 patients with active severe UC as defined by the Truelove and Witts criteria ( 8 men and 3 women; age range 20-63). Patients were required to have received at least 7 days of corticosteroid treatment and were permitted to be treated with immunomodulators excluding cyclosporine. Patients were randomly assigned to receive a single intravenous infusion of either placebo or infliximab at 5,10 , or $20 \mathrm{mg} / \mathrm{kg}$. Assessment of outcome was done at week 2 . The follow-up period was 4 weeks. The study was terminated prematurely because of slow enrolment.

Probert et al evaluated 43 patients with moderate UC based on ulcerative colitis symptom score (Schroeder et al 1987 ) and a sigmoidoscopy score (F/M ratio not given; age range 29-50.5) (Probert et al 2003). Patients were required to have been treated with corticosteroids for at least 1 week and were allowed to have received immunomodulators excluding cyclosporine if they were on a stable dose for at least 3 months prior to study enrollment. Patients were randomly assigned to receive an infusion of $5 \mathrm{mg} / \mathrm{kg}$ infliximab or placebo at week 0 and an identical infusion at week 2. Assessment of endpoints was done at week 6 , with follow-up for the following 30 days.

Järnerot et al evaluated 45 patients with moderate to severe UC according to the Seo index (Seo et al 1992) (24 men and 21 women; age range 19-61) (Järnerot et al 2005). Patients were required to have received at least 4 days of intravenous corticosteroids. No specific remark concerning prior treatment was defined in the inclusion criteria and 8 patients were treated with azathioprine at the time of enrollment. Patients were randomized to receive a single infusion of $5 \mathrm{mg} / \mathrm{kg} 4$ days after initiation of intravenous corticosteroids. Outcome measures were assessed 90 days after infliximab/placebo infusion.

Rutgeerts et al conducted the two largest studies to date that evaluated the efficacy of infliximab for induction and maintenance of remission in UC (Rutgeerts et al 2005). In the ACT1 study, 364 patients with moderate to severe colitis according to the Mayo Clinic score ( Schroeder et al 1987) with active colitis by sigmoidoscopy were recruited (222 men 142 women; age range 27-55). Patients were required to be on concurrent therapy with corticosteroids alone or with immunosuppressants. Patients were not required to be on concurrent treatment if they had previously not responded to these medications. Eligible patients were randomized to receive $5 \mathrm{mg} / \mathrm{kg}, 10 \mathrm{mg} / \mathrm{kg}$ of infliximab, or placebo in a $1: 1: 1$ ratio at weeks 0,2 , and 6 and then every 8 weeks for 46 weeks overall and were followed through week 54 . Outcome was measured at week 8, 30, and 54 .

ACT 2 was similar in design and included 364 patients (215 men and 149 women; age range 26-53). Patients received $5 \mathrm{mg} / \mathrm{kg}$ or $10 \mathrm{mg} / \mathrm{kg}$ infliximab or placebo in a 1:1:1 ratio at weeks 0,2 , and 6 and then every 8 weeks through week 22 and were followed through week 30 .

Interpretation of the results of the studies varied between the Cochrane and the systematic review. The systemic review by Gisbert et al (2007) evaluated short-term responses of infliximab vs placebo and found them to be $65 \%$ and $33 \%$, respectively (95\% confidence interval [CI] 61\%-69\% and $27 \%-38 \%$, respectively). The odds ratio (OR) for the response was $3.6(95 \%$ CI 2.67-4.95; p $<0.001)$, the number of treatments needed to achieve short-term response was 3 (95\% CI, 3-4) without significant heterogeneity between the studies. Short-term remission was achieved in 33\% (95\% CI 
$29 \%-37 \%)$ and $10 \%(95 \%$ CI, 6.4-14\%) in the infliximab and placebo groups, respectively. The OR for the remission was 4.56 (95\% CI 1.98-10.5; $\mathrm{p}<0.001)$ with NNT being $4(95 \%$ CI 3-6), results being significantly heterogeneous.

Long-term response was 53\% and 24\% in the infliximab and placebo groups (95\% CI 49\%-58\% and 19\%-29\%), respectively. The OR for the rsponse was 3.4 (95\% CI $2.52-$ 4.59; $\mathrm{p}<0.001)$ with NNT to achieve long term response being 3 (95\% CI 3-4) and no significant heterogeneity. Finally, long-term remission was achieved in $33 \%$ vs $14 \%$ in the infliximab and placebo groups ( $95 \%$ CI 29\%-37\% and 9\%-18\%), respectively. The OR for the remission was 2.72 (95\% CI 1.92-3.38; $\mathrm{p}<0.001)$ without significant heterogeneity and NNT to achieve long-term remission being 5 (95\% CI 4-7).

The Cochrane review (Lawson et al 2006) adopted a slightly different approach, describing the results in terms of reaching pre-defined end points.

\section{Clinical remission}

This endpoint was described in only 4 trials. The authors combined the ACT1 and ACT2 trials for the purpose of metaanalysis and reported the other two studies separately because of different outcome measures. Infliximab was effective for the induction of clinical remission at 8 weeks (relative risk [RR] 3.22, 95\% CI 2.18-4.16, NNT = 5). There was no significant difference in the results for the subgroup analysis of $5 \mathrm{mg} / \mathrm{kg}$ and $10 \mathrm{mg} / \mathrm{kg}$ of infliximab. The two small studies that reported this outcome showed a trend favoring infliximab but failed to reach statistical significance (Probert et al 2003; Järnerot et al 2005).

\section{Endoscopic remission/mucosal healing}

This outcome was also described in 4 studies. The two smaller studies did not reach statistical significance. The two larger studies were grouped for meta-analysis. Infliximab was effective in inducing endoscopic remission and mucosal healing at 8 weeks (RR 1.88, 95\% CI 1.54-2.28, NNT =4) without heterogeneity.

\section{Clinical response}

This endpoint was assessed in the ACT trials. infliximab effectively induced a clinical response (RR 1.99, 95\% CI 1.65$2.41, \mathrm{NNT}=4$ ) and there was moderate heterogeneity.

\section{Treatment success}

This outcome was assessed in only one small trial (Sands et al 2001). There was no statistical significance between infliximab and placebo.

\section{Colectomy}

This outcome was only described in one study (Järnerot et al 2005). There was a significant reduction in rates of colectomy with infliximab (RR, 95\% CI 0.22-0.78).

\section{Quality of life}

This outcome measure was assessed in one study (Probert et al 2003). Using a standard questionnaire (IBDQ), the authors did not find a statistically significant difference between infliximab and placebo in improving the quality of life.

\section{Safety}

Adverse events related to infliximab were generally mild and did not differ between the treatment and placebo groups. Patients in the infliximab groups developed headache, upper respiratory tract infection, pruritus, nephrolithiasis, and catheter insertion complication. Recently, concerns regarding the issue of severe infections including tuberculosis and the development of lymphoma after infliximab treatment were raised. In the ACT1 and ACT2 studies, one patient treated with infliximab in each trial developed serious infections, one a case of tuberculosis, and the other fatal histoplasmosis. Patients in the infliximab groups were more likely to develop auto-antibodies than patients in the placebo groups.

\section{Discussion and summary}

IBD is a chronic disease with a relapsing and remitting course. Its pathogenesis involves a dysregulated immune response to luminal antigens in patients with a genetic susceptibility. IBD comprises of two distinct clinical entities, UC and CD. Despite immunologic differences between them, TNF- $\alpha$, a pro-inflammatory cytokine, is hypothesized to play a key role in regulating the pathogenesis of both diseases. Therapy directed at blocking TNF- $\alpha$ action was shown to result in clinical improvement in patients with moderate to severe CD. In UC the role of TNF- $\alpha$ is less well established; however, there is mounting evidence that TNF- $\alpha$ gene polymorphisms are associated with disease extent and severity. Also, TNF- $\alpha$ levels were shown to be elevated in colonic tissue, feces, and serum of patients with UC and to correlate with disease severity. Initial studies evaluating the efficacy of anti-TNF- $\alpha$ treatment in moderate to severe UC showed relative success in inducing remission and in achieving clinical response. Most of these early studies were small and uncontrolled. Since then, 5 randomized placebo-controlled studies enrolling 827 patients assessed the efficacy of infliximab treatment in UC. The studies were heterogeneous. Three were small (less then 50 patients in each) and 2 were large (360 patients each). 
The studies utilized different outcome measures, making the results hard to compare. Two of the small studies did not show clinical benefit of infliximab (Probert et al 2003; Sands et al 2001), raising the possibility of a type II error. The other three studies all showed clinical benefit to infliximab (Järnerot et al 2005; Rutgeerts et al 2005); however, they did not assess the same endpoints. The smaller study by Järnerot et al (2005) showed a reduction in the rates of colectomy but did not look at remission. The study was small (45 patients) and the follow-up short (13 weeks). The two larger studies by Rutgeerts et al (2005) showed benefit in induction of remission, clinical response, discontinuing of corticosteroids, and mucosal healing in the groups treated with infliximab, but did not assess rates of colectomy. Based on the three studies and the two systematic reviews, infliximab is effective in inducing remission and improving clinical response in patients with moderate to severe UC. One study showed reduced rates of colectomy and two small studies did not find any benefit from infliximab treatment. The proportion of patients who reported adverse events were similar between the treatment and placebo groups. Most adverse events were mild. In the ACT1 and ACT2 studies, there was a slight increase in the number of serious infections, neurological complications, and lupus-like reactions in the infliximab groups with one fatal disseminated histoplasmosis.

Until recently, the only available medical treatment for patients with moderate to severe UC that failed conventional treatment was cyclosporine. Although infliximab is sometimes considered an alternative to cyclosporine, we believe that no competition should actually exist between the two drugs: infliximab has a slower mode of action and is used to benefit moderate to severe UC in ambulatory patients. Cyclosporine continues to be the only effective alternative to emergency colectomy in patients hospitalized for severe fulminant disease. The role of combined cyclosporine and infliximab has not been assessed and would increase the risk of profound immunosuppression. There are already reports of an increase in peri-operative infections in UC patients undergoing colectomy after treatment with both infliximab and cyclosporine.

The data reviewed here emphasizes the importance of establishing a uniform scoring system to UC similar to the one used in CD. Such a scoring system will enable appropriate comparison of clinical outcomes and assist in conducting adequate drug trials in UC. Special attention should be given to elucidating the mechanism of action of infliximab. Understanding the role of TNF- $\alpha$ and other mediators in the pathogenesis of UC will enhance our ability to design new drugs that will target key chemokines and cytokines regulators of the intestinal inflammatory process. Although the trials provide an answer to the question concerning the place of infliximab in the treatment of moderate to severe UC, several clinical questions remain unanswered. What is the role of infliximab in inducing remission in mild to moderate UC? What is the role of infliximab in maintenance of remission? Can infliximab be used for the treatment of extra-intestinal manifestations of UC? Is there a role for infliximab in the treatment of pouchitis? These questions will have to await the results of future well designed, double-blind, placebocontrolled trials.

\section{Abbreviations}

CRP, C-reactive protein; CI, confidence interval; IBD, inflammatory bowel disease, CD, Crohn's disease; UC, ulcerative colitis; TNF- $\alpha$, tumor necrosis factor- $\alpha$.

\section{Disclosures}

Neither author has any conflicts of interest to disclose.

\section{References}

Actis GC, Bruno M, Pinna-Pintor M, et al. 2002. Infliximab for treatment of steroid-refractory ulcerative colitis. Dig Liver Dis, 34:631.

Ahmad T, Armuzzi A, Neville M, et al. 2003. The contribution of human leucocyte antigen complex genes to disease phenotype in ulcerative colitis. Tissue Antigens, 62:527.

Argiles JM, Lopez-Soriano J, Busquets S, et al. 1997. Journey from cachexia to obesity by TNF. Faseb J, 11:743.

Armuzzi A, De Pascalis B, Lupascu A, et al. 2004. Infliximab in the treatment of steroid-dependent ulcerative colitis. Eur Rev Med Pharmacol Sci, 8:231.

Atreya R, Mudter J, Finotto S, et al. 2000. Blockade of interleukin 6 trans signaling suppresses $\mathrm{T}$-cell resistance against apoptosis in chronic intestinal inflammation: evidence in crohn disease and experimental colitis in vivo. Nat Med, 6:583.

Baugh JA, Bucala R. 2001. Mechanisms for modulating TNF alpha in immune and inflammatory disease. Curr Opin Drug Discov Devel, 4:635.

Baumgart DC, Sandborn WJ. 2007. Inflammatory bowel disease: clinical aspects and established and evolving therapies. Lancet, 369:1641.

Bazzoni F, Beutler B. 1996. The tumor necrosis factor ligand and receptor families. $N$ Engl J Med, 334:1717.

Bermejo F, Lopez-Sanroman A, Hinojosa J, et al. 2004. Infliximab induces clinical, endoscopic and histological responses in refractory ulcerative colitis. Rev Esp Enferm Dig, 96:94.

Binder V. 2004. Epidemiology of IBD during the twentieth century: an integrated view. Best Pract Res Clin Gastroenterol, 18:463.

Bischoff SC, Lorentz A, Schwengberg S, et al. 1999. Mast cells are an important cellular source of tumour necrosis factor alpha in human intestinal tissue. Gut, 44:643.

Black RA, Rauch CT, Kozlosky CJ, et al. 1997. A metalloproteinase disintegrin that releases tumour-necrosis factor-alpha from cells. Nature, 385:729.

Bouma G, Crusius JB, Garcia-Gonzalez MA, et al. 1999. Genetic markers in clinically well defined patients with ulcerative colitis (UC). Clin Exp Immunol, 115:294. 
Bouma G, Strober W. 2003. The immunological and genetic basis of inflammatory bowel disease. Nat Rev Immunol, 3:521.

Bouma G, Xia B, Crusius JB, et al. 1996. Distribution of four polymorphisms in the tumour necrosis factor (TNF) genes in patients with inflammatory bowel disease (IBD). Clin Exp Immunol, 103:391.

Braegger CP, Nicholls S, Murch SH, et al. 1992. Tumour necrosis factor alpha in stool as a marker of intestinal inflammation. Lancet, 339:89.

Breese EJ, Michie CA, Nicholls SW, et al. 1994. Tumor necrosis factor alpha-producing cells in the intestinal mucosa of children with inflammatory bowel disease. Gastroenterology, 106:1455.

Broome U, Lofberg R, Veress B, et al. 1995. Primary sclerosing cholangitis and ulcerative colitis: evidence for increased neoplastic potential. Hepatology, 22:1404.

Cao Q, Zhu Q, Wu ML, et al. 2006. Genetic susceptibility to ulcerative colitis in the Chinese Han ethnic population: association with TNF polymorphisms. Chin Med J (Engl), 119:1198.

Carter MJ, Lobo AJ, Travis SP. 2004. Guidelines for the management of inflammatory bowel disease in adults. Gut, 53, Suppl 5:V1.

Castro Fernandez M, Garcia Diaz E, Romero M, et al. 2003. [Treatment of steroid-refractory ulcerative colitis with infliximab]. Gastroenterol Hepatol, 26:54.

Cauwels A, Janssen B, Waeytens A, et al. 2003. Caspase inhibition causes hyperacute tumor necrosis factor-induced shock via oxidative stress and phospholipase A2. Nat Immunol, 4:387.

Chey WY, Hussain A, Ryan C, et al. 2001. Infliximab for refractory ulcerative colitis. Am J Gastroenterol, 96:2373.

Danese S, Semeraro S, Papa A, et al. 2005. Extraintestinal manifestations in inflammatory bowel disease. World J Gastroenterol, 11:7227.

D’Haens G, Lemmens L, Geboes K, et al. 2001. Intravenous cyclosporine versus intravenous corticosteroids as single therapy for severe attacks of ulcerative colitis. Gastroenterology, 120:1323.

D'Haens G, Sandborn WJ, Feagan BG, et al. 2007. A review of activity indices and efficacy end points for clinical trials of medical therapy in adults with ulcerative colitis. Gastroenterology, 132:763.

Elliott MJ, Maini RN, Feldmann M, et al. 1993. Treatment of rheumatoid arthritis with chimeric monoclonal antibodies to tumor necrosis factor alpha. Arthritis Rheum, 36:1681

Elson CO, Cong Y, McCracken VJ, et al. 2005. Experimental models of inflammatory bowel disease reveal innate, adaptive, and regulatory mechanisms of host dialogue with the microbiota. Immunol Rev, 206:260.

Gisbert JP, Gonzalez-Lama Y, Mate J. 2007. Systematic review: Infliximab therapy in ulcerative colitis. Aliment Pharmacol Ther, 25:19.

Gornet JM, Couve S, Hassani Z, et al. 2003. Infliximab for refractory ulcerative colitis or indeterminate colitis: an open-label multicentre study. Aliment Pharmacol Ther, 18:175.

Guy-Grand D, DiSanto JP, Henchoz P, et al. 1998. Small bowel enteropathy: role of intraepithelial lymphocytes and of cytokines (IL-12, IFN-gamma, TNF) in the induction of epithelial cell death and renewal. Eur J Immunol, 28:730.

Hanai H, Watanabe F, Yamada M, et al. 2004. Correlation of serum soluble TNF-alpha receptors I and II levels with disease activity in patients with ulcerative colitis. Am J Gastroenterol, 99:1532.

Hanauer SB, Cohen RD, Becker RV 3rd, et al. 1998. Advances in the management of Crohn's disease: economic and clinical potential of infliximab. Clin Ther, 20:1009.

Ina K, Itoh J, Fukushima K, et al. 1999. Resistance of Crohn's disease T cells to multiple apoptotic signals is associated with a Bcl-2/Bax mucosal imbalance. J Immunol, 163:1081.

Iwakura Y, Ishigame H. 2006. The IL-23/IL-17 axis in inflammation. J Clin Invest, 116:1218.

Jacobsohn DA, Vogelsang GB. 2004. Anti-cytokine therapy for the treatment of graft-versus-host disease. Curr Pharm Des, 10:1195.

Järnerot G, Hertervig E, Friis-Liby I, et al. 2005. Infliximab as rescue therapy in severe to moderately severe ulcerative colitis: a randomized, placebo-controlled study. Gastroenterology, 128:1805.
Jung HC, Eckmann L, Yang SK, et al. 1995. A distinct array of proinflammatory cytokines is expressed in human colon epithelial cells in response to bacterial invasion. $J$ Clin Invest, 95:55.

Knight DM, Trinh H, Le J, et al. 1993. Construction and initial characterization of a mouse-human chimeric anti-TNF antibody. Mol Immunol, 30:1443.

Kohn A, Prantera C, Pera A, et al. 2002. Anti-tumour necrosis factor alpha (infliximab) in the treatment of severe ulcerative colitis: result of an open study on 13 patients. Dig Liver Dis, 34:626.

Komatsu M, Kobayashi D, Saito K, et al. 2001. Tumor necrosis factor-alpha in serum of patients with inflammatory bowel disease as measured by a highly sensitive immuno-PCR. Clin Chem, 47:1297.

Korzenik JR, Podolsky DK. 2006. Evolving knowledge and therapy of inflammatory bowel disease. Nat Rev Drug Discov, 5:197.

Kountouras J, Zavos C, Chatzopoulos D. 2005. Anti-tumor necrosis factor therapy for ulcerative colitis. Gastroenterology, 129:1138.

Lawson MM, Thomas AG, Akobeng AK. 2006. Tumour necrosis factor alpha blocking agents for induction of remission in ulcerative colitis. Cochrane Database Syst Rev, 3:CD005112.

Lennard-Jones JE, Ritchie JK, Hilder W, et al. 1975. Assessment of severity in colitis: a preliminary study. Gut, 16:579.

Lichtenstein GR, Abreu MT, Cohen R, et al. 2006. American Gastroenterological Association Institute medical position statement on corticosteroids, immunomodulators, and infliximab in inflammatory bowel disease. Gastroenterology, 130:935.

Lichtiger S, Present DH, Kornbluth A, et al. 1994. Cyclosporine in severe ulcerative colitis refractory to steroid therapy. $N$ Engl J Med, 330:1841.

Ljung T, Karlen P, Schmidt D, et al. 2004. Infliximab in inflammatory bowel disease: clinical outcome in a population based cohort from Stockholm County. Gut, 53:849.

Loftus EV Jr, Silverstein MD, Sandborn WJ, et al. 2000. Ulcerative colitis in Olmsted County, Minnesota, 1940-1993: incidence, prevalence, and survival. Gut, 46:336.

MacDonald TT, Hutchings P, Choy MY, et al. 1990. Tumour necrosis factor-alpha and interferon-gamma production measured at the single cell level in normal and inflamed human intestine. Clin Exp Immunol, 81:301.

Murch SH, Braegger CP, Walker-Smith JA, et al. 1993. Location of tumour necrosis factor alpha by immunohistochemistry in chronic inflammatory bowel disease. Gut, 34:1705.

Nilsen EM, Johansen FE, Jahnsen FL, et al. 1998. Cytokine profiles of cultured microvascular endothelial cells from the human intestine. Gut, 42:635

Ochsenkuhn T, Sackmann M, Goke B. 2004. Infliximab for acute, not steroid-refractory ulcerative colitis: a randomized pilot study. Eur $J$ Gastroenterol Hepatol, 16:1167.

Old LJ. 1985. Tumor necrosis factor (TNF). Science, 230:630.

Papadakis KA, Targan SR. 2000. Role of cytokines in the pathogenesis of inflammatory bowel disease. Annu Rev Med, 51:289.

Park H, Li Z, Yang XO, et al. 2005. A distinct lineage of CD4 T cells regulates tissue inflammation by producing interleukin 17. Nat Immunol, 6:1133.

Peppelenbosch MP, van Deventer SJ. 2004. T cell apoptosis and inflammatory bowel disease. Gut, 53:1556.

Plevy SE, Landers CJ, Prehn J, et al. 1997. A role for TNF-alpha and mucosal T helper-1 cytokines in the pathogenesis of Crohn's disease. J Immunol, 159:6276.

Podolsky DK. 2002. Inflammatory bowel disease. $N$ Engl J Med, 347:417.

Probert CS, Hearing SD, Schreiber S, et al. 2003. Infliximab in moderately severe glucocorticoid resistant ulcerative colitis: a randomised controlled trial. Gut, 52:998.

Remicade package insert (Centocor-US), N., Rec 9/24/98.

Ruiz P, San Salvador P, Ortiz de Zarate J, et al. 2004. [Infliximab as treatment for a severe outbreak of ulcerative colitis]. Gastroenterol Hepatol, 27:430. 
Rutgeerts P, Sandborn WJ, Feagan BG, et al. 2005. Infliximab for induction and maintenance therapy for ulcerative colitis. $N$ Engl J Med, $353: 2462$

Sandborn WJ, Hanauer SB. 1999. Antitumor necrosis factor therapy for inflammatory bowel disease: a review of agents, pharmacology, clinical results, and safety. Inflamm Bowel Dis, 5:119.

Sands BE, Kaplan GG. 2007. The role of TNFalpha in ulcerative colitis. $J$ Clin Pharmacol, 47:930.

Sands BE, Tremaine WJ, Sandborn WJ, et al. 2001. Infliximab in the treatment of severe, steroid-refractory ulcerative colitis: a pilot study. Inflamm Bowel Dis, 7:83.

Schroeder KW, Tremaine WJ, Ilstrup DM. 1987. Coated oral 5-aminosalicylic acid therapy for mildly to moderately active ulcerative colitis. A randomized study. N Engl J Med, 317:1625.

Seo M, Okada M, Yao T, et al. 1992. An index of disease activity in patients with ulcerative colitis. Am J Gastroenterol, 87:971.

Shen C, de Hertogh G, Bullens DM, et al. 2007. Remission-inducing effect of anti-TNF monoclonal antibody in TNBS colitis: mechanisms beyond neutralization? Inflamm Bowel Dis, 13:308.

Shibolet, O, Regushevskaya E, Brezis M, et al. 2005. Cyclosporine A for induction of remission in severe ulcerative colitis. Cochrane Database Syst Rev:CD004277.

Siddiqui MA, Scott LJ. 2005. Infliximab: a review of its use in Crohn's disease and rheumatoid arthritis. Drugs, 65:2179.

Soetikno RM, Lin OS, Heidenreich PA, et al. 2002. Increased risk of colorectal neoplasia in patients with primary sclerosing cholangitis and ulcerative colitis: a meta-analysis. Gastrointest Endosc, 56:48.

Spoettl T, Hausmann M, Klebl F, et al. 2007. Serum soluble TNF receptor I and II levels correlate with disease activity in IBD patients. Inflamm Bowel Dis, 13:727.

Strober W, Fuss IJ, Blumberg RS. 2002. The immunology of mucosal models of inflammation. Annu Rev Immunol, 20:495.
Su C, Salzberg BA, Lewis JD, et al. 2002. Efficacy of anti-tumor necrosis factor therapy in patients with ulcerative colitis. Am J Gastroenterol, 97:2577.

Tilg H, Moschen A, Kaser A. 2007. Mode of function of biological antiTNF agents in the treatment of inflammatory bowel diseases. Expert Opin Biol Ther, 7:1051.

Truelove SC, Witts LJ. 1955. Cortisone in ulcerative colitis; final report on a therapeutic trial. $\mathrm{Br} \mathrm{Med} J, 2: 1041$

Van Deventer SJ. 1997. Tumour necrosis factor and Crohn's disease. Gut, 40:443.

van Dullemen HM, van Deventer SJ, Hommes DW, et al. 1995. Treatment of Crohn's disease with anti-tumor necrosis factor chimeric monoclonal antibody (cA2). Gastroenterology, 109:129.

van Heel DA, Udalova IA, De Silva AP, et al. 2002. Inflammatory bowel disease is associated with a TNF polymorphism that affects an interaction between the OCT1 and NF(-kappa)B transcription factors. Hum Mol Genet, 11:1281.

Vassalli P. 1992. The pathophysiology of tumor necrosis factors. Annu Rev Immunol, 10:411.

Watkins PE, Warren BF, Stephens S, et al. 1997. Treatment of ulcerative colitis in the cottontop tamarin using antibody to tumour necrosis factor alpha. Gut, 40:628.

Wilson AG, de Vries N, Pociot F, et al. 1993. An allelic polymorphism within the human tumor necrosis factor alpha promoter region is strongly associated with HLA A1, B8, and DR3 alleles. J Exp Med, 177:557.

Winther KV, Jess T, Langholz E, et al. 2003. Survival and cause-specific mortality in ulcerative colitis: follow-up of a population-based cohort in Copenhagen County. Gastroenterology, 125:1576.

Xavier RJ, Podolsky DK. 2007. Unravelling the pathogenesis of inflammatory bowel disease. Nature, 448:427. 